\title{
southeastern Italy
}

\section{Andrea Pieroni $^{i *}$ (1) and Valentina Cattero ${ }^{1,2}$ (1)}

Received: September 24, 2018

Accepted: December 18, 2018

\begin{abstract}
Bio-cultural and historical aspects of the "wild plant" portion of the Mediterranean Diet are still very much understudied despite the large number of bio-medical studies on the topic. The current gastronomic ethnobotanical and ethnolinguistic field study focused on the wild leafy vegetables used among Greek populations in SE Italy and NE Greece. A total of 52 folk taxa, corresponding to 58 wild botanical species, were recorded. The frequency of consumption of these wild ingredients was higher in NE Greece than in SE Italy, although approximately one-third of the recorded wild taxa overlapped in the two study sites. Most of these common species were designated by cognates, having in most cases a clear Greek origin, while one-third of the recorded wild vegetables in SE Italy were also used by another Greek diaspora living in SW Italy. The majority of the original Greek wild vegetables are synanthropic weeds. It is likely that the culinary uses of these species originated in the Near East during the post-Neolithic period before they migrated west to Italy and the Mediterranean Basin via Greece and Greek diasporas.
\end{abstract}

Keywords: ethnobotany, Greece, Italy, Mediterranean Diet, wild vegetables

\section{Introduction}

Naska \& Trichopoulou (2014) defined the Mediterranean Diet as a dietary system abundant in plant foods, fresh fruits, olive oil as the principal source of fat, dairy products as well as fish and poultry consumed in low to moderate amounts, zero to four eggs consumed weekly, red meat consumed in low amounts, and wine consumed in low to moderate amounts, normally with meals.

While a remarkable spectrum of bio-scientific literature has stressed the health benefits of this diet (Menotti \& Puddu 2015, and references therein), the cultural and historical dynamics of the long journey through which this dietary system developed as a bio-cultural complex are often neglected (Ferrari \& Rapezzi 2011). This is somewhat surprising given that food is primarily a cultural issue (Montanari 2006). In addition, the Mediterranean Diet has been recognized by UNESCO in cultural terms as a part of the Intangible Cultural Heritage of Humanity (UNESCO 2013).

Only a few studies have analysed in detail the nutraceutical properties of neglected, non-cultivated ingredients of the Mediterranean Diet (Trichopoulou et al. 2000; Local Food-Nutraceuticals Consortium 2005; Conforti et al. 2011; 2012; Vasilopoulou \& Trichopoulou 2011; Romojaro et al. 2013; Marrelli et al. 2014) and very little research has been done on the historical and cultural (anthropological and/or ethnolinguistic) aspects of the

1 University of Gastronomic Sciences, I-12060, Pollenzo, Cuneo, Italy

2 Institut sur la Nutrition et les Aliments Fonctionnels, Université Laval, G1V 0A6, Québec, Québec, Canada

* Corresponding author: a.pieroni@unisg.it 
'hidden part' of this dietary system (Pieroni et al. 2002; 2005; Nebel et al. 2006; di Tizio et al. 2012; Marouf et al. 2015; Cucinotta \& Pieroni 2018).

Moreover, some ethnobotanical studies have assessed the wild food vegetables in the local daily diets of coastal and/or rural Mediterranean areas of Spain, Southern Italy, Turkey, Croatia and Herzegovina (Tardío et al. 2005; Della et al. 2006; Dogan 2012; Łuczaj et al. 2013; Biscotti \& Pieroni 2015; Łuczaj \& Dolina 2015; Licata et al. 2016; Rigat et al. 2016; Geraci et al. 2018). However, such research in the Near East and Northern Africa is still extremely rare (Abdalla 2004; Marouf et al. 2015). Very few methodologically rigorous food ethnobotanical field studies on Greece have been published in international journals even though this country has been the core area for many studies concerning the Mediterranean Diet. In addition, the diversity of the wild vegetables that are gathered and sold in local farmers' markets in a few Greek regions, most notably Crete, is extraordinary (A Pieroni unpubl. res.).

Cross-cultural studies comparing the wild food plant ingredients of different ethnic or linguistic groups in the Mediterranean and the Near East have been even more scarce (Leonti et al. 2006; Ghirardini et al. 2007; Santayana et al. 2007; Hadjichambis et al. 2008; Pieroni et al. 2018). Such studies are needed for assessing differences in the ancient practice of gathering plant foods from the wild and the socio-cultural variables that might have influenced the use of specific wild ingredients in the Mediterranean Diet. Unfortunately, the number of people in Southern Europe with this kind of traditional knowledge has been decreasing since the 1960s. Recently, the wild plants gathered by younger generations have reflected a combination of some 'traditional' customs and new trends managed by urban 'foodies' (Euczaj et al. 2012), which are often reinforced by social media.

The goal of the present ethnobotanical study was to perform an ethnolinguistic and ethnobotanical analysis of the wild vegetables gathered and consumed by a littleknown Greek diaspora in Southern Italy: the Griko people of Grecia Salentina, the Salento area, Southern Apulia, SE Italy. In order to research the "roots" of this South Italian Greek diaspora, and given the lack of recent reliable Greek ethnobotanical data, a comparative sample was drawn from rural NE Greece.

The objectives of the current study were therefore the following: to analyse the wild vegetables still gathered and consumed by the Griko people of SE Italy during the spring, which included recording their local names, frequency of use and quotation, gathering periods, traditional culinary preparations and the health benefits attributed to them by local residents; to evaluate the presence of the abovementioned phenomena among Greeks living in rural areas of NE Greece; to compare the data from the two study sites and that arising from previous ethnobotanical studies on South Italian and Greek wild food plants, with special attention to a spring 2002-2003 study on the Graecanic people, the other Greek diaspora living in SW Italy (Nebel et al. 2006); and to analyse the linguistic aspects of the data (folk names of the plants) to uncover the Greek origins of the Griko folk phytonyms and, where possible, ancient uses of wild food ingredients.

\section{Materials and methods}

\section{The Italian study site}

Apulia is one of the southern Italian regions colonised by Greeks in ancient times as a result of the growth of the Greek population and the emergent interest in trade. Beginning in the 8th century B.C., the immigrants founded colonies in Apulia, Basilicata, Calabria, Campania and Sicily, and

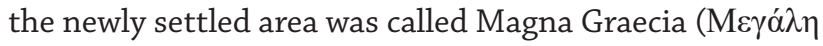
E $E \lambda \lambda \alpha$ ś) to proclaim the greatness of the colonies compared to that of the motherland. Despite its significant number of colonies, Sicily was not considered part of Magna Graecia until Roman historians included it in their definition. In Southern Apulia, in the areas of the former Greek colonies (e.g. Tarentum, present-day Taranto; or Callipolis, presentday Gallipoli) one can find the linguistic island of Grecia Salentina, a community of nine municipalities in the province of Lecce. The language in these municipalities is Griko, a neo-Greek dialect peculiar to this area of Salento. It represents one of the communities recognised by the Italian government as a linguistic minority. The origin of this long-lasting language is controversial. According to the German philologist Gerhard Rohlfs, Griko may represent continuity from the ancient colonies of Magna Graecia (Rohlfs 1967). In contrast, Italian research groups have concluded that these communities more likely derive from a later immigration, during the medieval period, of people who had previously emigrated from the Byzantine Empire during the 6 th century A.D. The rationale for this assertion is based on the similarities between the Griko dialect and Modern Greek (Parlangeli 1989).

More recently, the common theory has been that these communities have been developing through consecutive waves of migration, with the medieval communities strengthening the pre-existing ancient ones. Indeed, the introduction of Greek Orthodox Christian practices by the Byzantines contributed to the establishment of Greek customs. After invasion by the Normans and subsequent invasions by the Swabians, the Capetian House of Anjou and the Crown of Aragon, the eastern culture continued to survive despite the beliefs of these various conquerors. In the 16th century A.D., the Council of Trent's declaration of an end to practices, including those of Greek origin, other than the official Latin ones led to the first significant contraction of the area. Whereas during the 15 th century the entire area between Gallipoli and Otranto, which consisted of 24 villages, was Grika, only 13 villages had survived by the end of the 18th century. Today, no more than 11 are 
left, and only nine municipalities retain the language. Griko is not a written language; it is transmitted orally through the generations. Ultimately, with the unification of Italy in 1861, standard (Tuscan) Italian became the official language of the nation; and later, after World War II, social changes induced by industrialization and urbanization cast Griko, as well as other minority languages throughout Italy, as a 'language of shame' (mas èkanne vergògna, Pellegrino 2016). For the same people living in Grecia, the language came to connote a lack of sophistication unlike Italian, which was considered the language of progress and modernity. When Griko was discovered by scholars during the 19th century, it was already regarded as a dying language (Pellegrino 2016). Nowadays only elderly community members in Grecia Salentina can speak Griko, and Griko is considered by UNESCO as a severely endangered language (Moseley 2010)

The local economy is still based on agriculture, with significant legume production; and the harvest and consumption of wild greens are quite common throughout Salento, especially in Grecìa.

\section{The Greek study site}

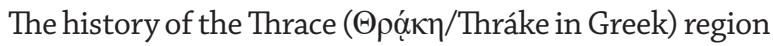
predates the Greek and Roman periods. Several of the tribes that were living in the territory without any organised or centralised power were called Thracians by the Greeks. Later, the area began to be Hellenised, even before the Peloponnesian war (431-404 B.C.), when the Athenians established some colonies along the shore. In 168 B.C., after the surrender of Macedonia to the Romans, Thrace also fell under Roman control, becoming a tributary to the empire until 46 A.D. when it became Romana Provincia Thracia, a province of the Roman Empire (Samsaris 1988). During Roman domination the area became more urbanised. This process actually favoured Hellenisation, rather than Latinisation, of the area. After the collapse of the Western Roman Empire, the eastern empire governed by Byzantium (at that time Constantinople) enjoyed a period of prosperity until the 8th Century, when a conflict with the Bulgarians began over the control of Thrace. With the conquest of the area by the Ottomans and the fall of the Eastern Roman Empire in 1352, five centuries of Muslim control thus began. The territory was circumscribed in the north by the Balkans, in the south by the Rhodope Mountains and the Aegean Sea, and in the east by the Black Sea. Thrace therefore has three distinct areas: Bulgarian, the biggest, in the north; Turkish in the east; and Greek in the west.

In Greek Thrace, the field study focused on the former district of Evros, named for the river that separates it from European Turkey, namely Eastern Thrace. This is likely the area from which the emigrants who settled in the Greek part of Salento, Italy, left during the height of the Byzantine Empire. Western Thrace is also home to a large number of the Muslim minorities of Greece. This can be traced back to the 1923 Greek-Turkish population exchange established by the Treaty of Lausanne. The small Muslim population subsequently gained status as an officially recognized minority and today accounts for one-third of the population of Western Thrace. About half of these Muslims are of Turkish origin, and about half are Bulgarian-speaking Pomaks.

The economy of Western Thrace is based mainly on agriculture, although recently there has been some industrial development. Wine and olive oil are still the leading products. Tobacco, once very important, has been partly replaced by sunflower, corn, rice and wheat. The historical cultivation and processing of silk, which dates back to the Byzantine era, still survives in Soufli. Tourism has started to develop in this region, which had always been one of the more neglected parts of the nation. Summer beaches as well as the Rhodope Mountains have recently been attracting a consistent number of tourists during winter. Nevertheless, the rural area remains almost untouched by this phenomenon, and thus the countryside has been preserved.

\section{Fieldwork and data collection}

The study was conducted during spring 2016 through interviews with 60 participants from villages in SE Italy and NE Greece (see Fig. 1). Thirty (30) interviewees (17 women and 13 men) were from SE Italy, and 30 (23 women and 7 men) were from NE Greece. The average age was 64 years. The participants were community members who still had strong ties to rural life and worked, at least part-time, as farmers or shepherds.

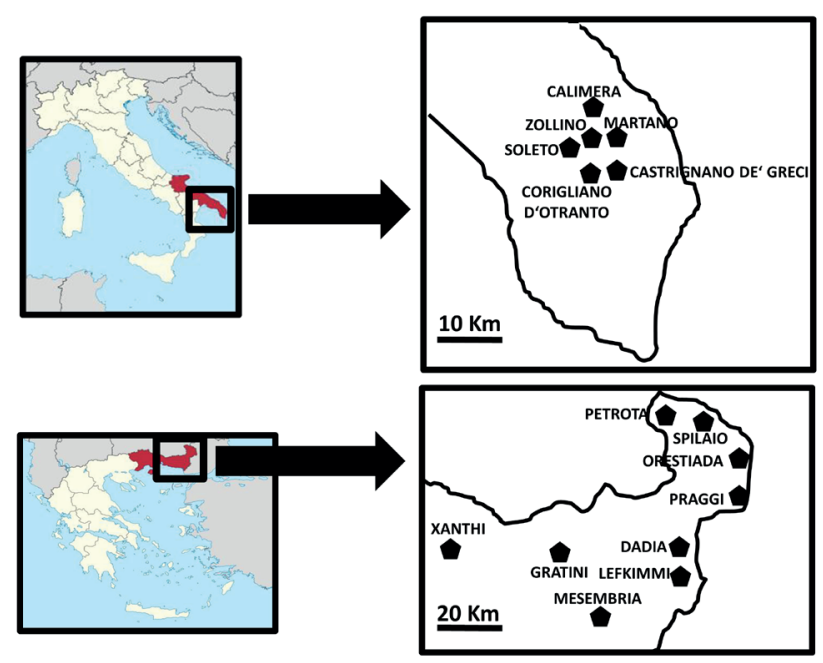

Figure 1. Study sites and visited villages.

Semi-structured interviews were administered in Italian and Greek with the help of a translator. Specifically, the participants were asked about: (a) the local name(s) of each 
wild plant used in the community, (b) the gatherers and the periods during which the plants were collected, (c) the plant parts used in the kitchen, (d) specific types of culinary preparation, (e) the frequency of use during the spring months, and (f) the perceived nutraceutical properties of each wild vegetable.

Before and during the field study, the International Society of Ethnobiology Code of Ethics was followed (ISE 2008). Voucher specimens were collected during a previous field study (Biscotti \& Pieroni 2015) and identified on the basis of Pignatti's Flora d'Italia (Pignatti 1982), Flora Europaea (Tutin et al. 2001), the most recent checklist of Greek vascular plants (Dimopoulos et al. 2016), and two local (food) floristic guides (Stavridakis 2006; Accogli \& Medagli 2014).

The plant nomenclature was standardized to The Plant List database (2018), while the family assignments followed the Angiosperm Phylogeny Group IV (Stevens 2017). The fungal nomenclature was based on the Index Fungorum Partnership (2018). The folk names were transcribed in accordance with the rules of standard Italian for Griko plant names and standard Greek for Greek plant names. The Greek phytonyms were later transliterated into the Latin alphabet.

\section{Data analysis}

The data collected in SE Italy were compared with: (a) the most extensive reviews conducted in the previous two decades in the same region of Apulia (Biscotti 2012; Ditonno \& Lamusta 2015; Biscotti et al. 2018); (b) other Southern Italian reviews or recent food ethnobotanical field studies (Lentini \& Venza 2007; Biscotti \& Pieroni 2015; Licata et al. 2016; Quave \& Saitta, 2016; Geraci et al. 2018); and (c) a previous study that was conducted in spring 2002-2003 among the Greek diaspora in Italy, i.e. the Graecanic people of Calabria, SW Italy (Nebel et al. 2006).

The Greek records were compared with the most extensive ethnobotanical field studies previously conducted in Greek speaking areas (Cyprus; Della et al 2006). The comparisons included the recorded wild plant species locally gathered and consumed as leafy vegetables (excluding mushrooms), and the findings were then illustrated in Venn diagrams in order to highlight commonalities and differences.

\section{Results}

Table 1 lists the vegetables that were recorded as still being gathered and consumed in the two study areas. In total, 52 folk taxa corresponding to 61 species were recorded, of which 58 referred to wild plants, one to a mushroom and two to cultivated vegetables whose uses diverged from the known "usual" culinary customs. The taxa designated by linguistic cognates in the two study areas are presented in bold type.
Figure 2 presents a comparison of the frequency of consumption in the South Italian and the Greek study sites. As indicated, the frequency of consumption of wild vegetables was higher in Greece than in Southern Italy.
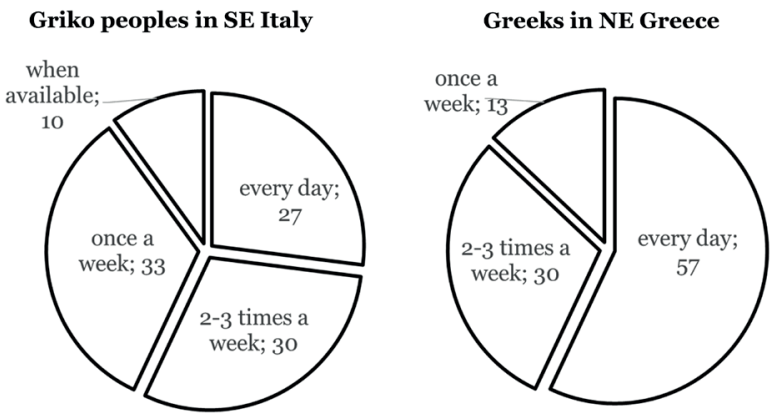

Figure 2. Frequency of consumption of recorded wild vegetables in the study sites.

Figure 3 illustrates the overlap between the folk taxa of the wild vegetables only (excluding the one mushroom and two cultivated plants) of the Italian and Greek study sites. One-third ( $\mathrm{n}=17)$ of the recorded taxa were common to both study sites, and the majority of these common folk taxa $(\mathrm{n}=12)$ were designated by the same or similar (cognate) Greek folk names.

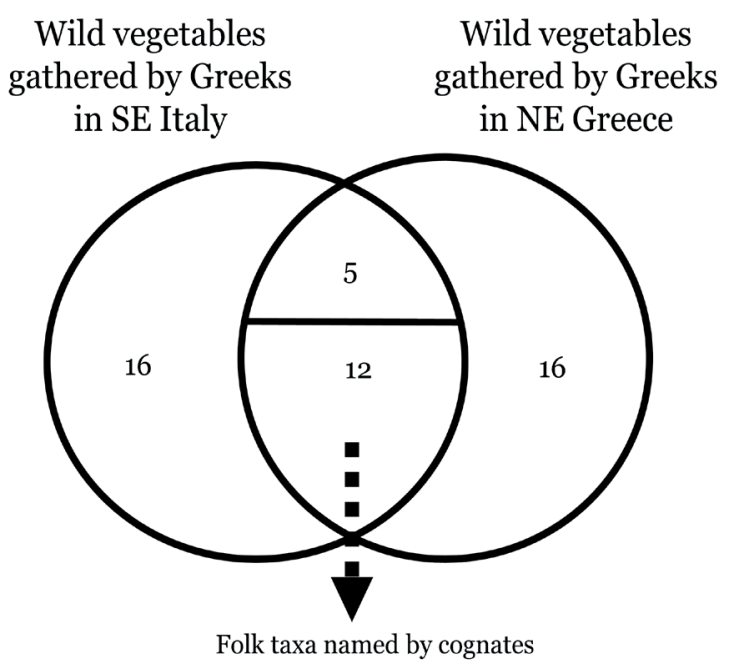

Figure 3. Comparison of the wild vegetables (folk taxa) gathered in SE Italy and NE Greece.

Figure 4 presents a comparison of the South Italian data collected from the Griko people and those collected in 2002-2003 in the Greek diaspora in SW Italy (Nebel et al. 2006). Approximately one-third $(\mathrm{n}=15)$ of the recorded wild vegetables (botanical species) were the same in the two studies, and one-half of the commonly gathered species $(n=8)$ were designated by cognates.

A Greek origin of the folk plant names is especially clear for the following botanical genera (Dioscorides 1959; Rohlfs 
Table 1. Wild vegetables gathered and collected in the study areas. The taxa designated by linguistic cognates in the two study areas are presented in bold type.

\begin{tabular}{|c|c|c|c|c|c|c|}
\hline Botanical taxon (-taxa) and family & Local name(s) & Used plant parts & Gathering time & Traditional culinary use(s) & $\begin{array}{c}\text { Frequency of } \\
\text { quotation }\end{array}$ & $\begin{array}{l}\text { Perceived nutraceutical } \\
\text { property (-ies) }\end{array}$ \\
\hline $\begin{array}{l}\text { Allium schoenoprasum L. } \\
\text { (Amaryllidaceae) }\end{array}$ & Schinopraso (GR) & Leaves & All year long & Raw & $\mathrm{R}$ & None \\
\hline $\begin{array}{l}\text { Amaranthus viridis } \mathrm{L} . \\
\text { Amaranthaceae) }\end{array}$ & Vlita (GR) & Young leaves & March-May & Boiled; boiled and then in salty pies & $\mathrm{VC}$ & None \\
\hline $\begin{array}{l}\text { Ammi majus L. } \\
\text { (Apiaceae) }\end{array}$ & Mùrlu, Mùruddhu (IT) & Basal rosettes & March-May & $\begin{array}{l}\text { Boiled in mixtures; boiled and fried, also as a } \\
\text { dressing on home-made pasta }\end{array}$ & $\mathrm{VC}$ & Against skin problems \\
\hline $\begin{array}{l}\text { Anthriscus cerefolium (L.) Hoffm } \\
\text { (Apiaceae) }\end{array}$ & Myroni (GR) & Leaves & March-May & $\begin{array}{l}\text { Seasoning (esp. soup made with lamb meat and } \\
\text { lettuce) }\end{array}$ & $\mathrm{R}$ & None \\
\hline $\begin{array}{l}\text { Asparagus acutifolius L. } \\
\text { (Asparagaceae) }\end{array}$ & $\begin{array}{l}\text { Agria sparaggia, Sparaggi, } \\
\text { Zaparoynes (GR); Spàraci, } \\
\text { Spàrasciu (IT) }\end{array}$ & Turions & March-April & $\begin{array}{l}\text { Omelettes and roasted (GR); boiled, omelettes, and } \\
\text { risotto (IT) }\end{array}$ & $\mathrm{VC}$ & $\begin{array}{l}\text { Diuretic and strengthening } \\
\text { (GR \& IT); against diabetes } \\
\text { (IT) }\end{array}$ \\
\hline $\begin{array}{l}\text { Beta vulgaris } \mathrm{L} \text {. } \\
\text { (Amaranthaceae) }\end{array}$ & Agrioseskoylo (GR) & $\begin{array}{l}\text { Young tender } \\
\text { leaves }\end{array}$ & March-June & Boiled and in salty pies & C & None \\
\hline $\begin{array}{l}\text { Borago officinalis L. } \\
\text { (Boraginaceae) }\end{array}$ & Burraccia, Burracciu (IT) & Aerial parts & January-April & $\begin{array}{l}\text { Boiled in mixtures; omelettes, shortly boiled and } \\
\text { then fried, filling for deep fried dough pieces } \\
\text { ("pittule ripiene", consumed esp. during Christmas, } \\
\text { the feast of Immaculate Conception/8th December, } \\
\text { and during Carnival) }\end{array}$ & , & None \\
\hline $\begin{array}{l}\text { Capparis spinosa L. } \\
\text { (Capparacaceae) }\end{array}$ & Chàpparu, Chiappru (IT) & $\begin{array}{l}\text { Leaves, young } \\
\text { shoots, and } \\
\text { flower buds }\end{array}$ & March-July & $\begin{array}{l}\text { Leaves and young shoots: boiled in mixtures. } \\
\text { Buds: pickled }\end{array}$ & C & None \\
\hline Capsella bursa-pastoris (L.) Medik. & $\begin{array}{l}\text { Agriokardamoyda (GR); Erba } \\
\text { raparina (IT) }\end{array}$ & Basal rosettes & February-April & Boiled (GR); boiled in mixtures and omelettes (IT) & $\mathrm{R}$ & Anti-haemorrhagic (IT) \\
\hline $\begin{array}{l}\text { Calystegia sepium (L.) R. Br., Convolvulus } \\
\text { arvensis L., and C. prostrates Forssk } \\
\text { (Convulvolaceae) }\end{array}$ & $\begin{array}{l}\text { Ntefeses, Sarmasikia, Sovleka, } \\
\text { Voyrlida (GR) }\end{array}$ & Leaves & May-June & $\begin{array}{l}\text { Raw (as a digestive "medicinal food"); soups (with } \\
\text { corn flour); filling for salty pies (in combination } \\
\text { with Lactuca and Papaver spp.) }\end{array}$ & $\mathrm{VC}$ & Digestive \\
\hline $\begin{array}{l}\text { Carduus nutans L., C. pycnocephalus } \\
\text { L., and Galactities tomentosa Moench } \\
\text { (Asteraceae) }\end{array}$ & $\begin{array}{l}\text { Agrio gaidoyragkatho (GR); } \\
\text { Cardu, Carduni (IT) }\end{array}$ & $\begin{array}{l}\text { Basal rosettes, } \\
\text { leaves, and stems } \\
\text { (after removing } \\
\text { thorns) }\end{array}$ & October-April & $\begin{array}{l}\text { Raw and boiled (GR); soups; boiled and then stir- } \\
\text { fried and eventually cooked in the oven (IT) }\end{array}$ & $\begin{array}{l}\mathrm{R}(\mathrm{GR}) ; \mathrm{VC} \\
\quad \text { (I) }\end{array}$ & Strengthening (IT) \\
\hline
\end{tabular}


Wild vegetables do not lie: Comparative gastronomic ethnobotany and ethnolinguistics on the Greek traces of the Mediterranean Diet of southeastern Italy

Table 1. Cont.

\begin{tabular}{|c|c|c|c|c|c|c|}
\hline Botanical taxon (-taxa) and family & Local name(s) & Used plant parts & Gathering time & Traditional culinary use(s) & $\begin{array}{c}\text { Frequency of } \\
\text { quotation }\end{array}$ & $\begin{array}{l}\text { Perceived nutraceutical } \\
\text { property (-ies) }\end{array}$ \\
\hline $\begin{array}{l}\text { Celtis australis } \mathrm{L} \text {. } \\
\text { (Cannabaceae) }\end{array}$ & Spaccasassi, Erva rustica (IT) & Leaves & March-May & Boiled in mixtures & $\mathrm{R}$ & $\begin{array}{l}\text { Against kidney stones and } \\
\text { gallstones }\end{array}$ \\
\hline $\begin{array}{l}\text { Celosia argentea } \mathrm{L} \text {. } \\
\text { (Amaranthaceae) }^{*}\end{array}$ & Leiri toy peteinoy (GR) & Tender leaves & March-May & Boiled and in salty pies & $\mathrm{R}$ & None \\
\hline $\begin{array}{l}\text { Chenopodium album L. } \\
\text { (Amaranthaceae) }\end{array}$ & Louvoydia, Limpoydia (GR) & Young leaves & March-May & $\begin{array}{l}\text { Boiled, salty pies (sometimes with dough made } \\
\text { from lard and flour) }\end{array}$ & $\mathrm{VC}$ & None \\
\hline $\begin{array}{l}\text { Chenopodium bonus- henricus L. } \\
\text { (Amaranthaceae) }\end{array}$ & Agrio spanáki (GR) & Basal rosettes & March-May & Boiled and salty pies & $\mathrm{VC}$ & None \\
\hline $\begin{array}{l}\text { Cichorium intybus L. } \\
\quad \text { (Asteraceae) }\end{array}$ & $\begin{array}{l}\text { Agratsida, Kichorio, } \\
\text { Pikroradiko, Radiko (GR); } \\
\text { Cicureddha (IT) }\end{array}$ & Basal rosettes & October-April & $\begin{array}{l}\text { Consumed raw on bread, boiled, boiled and stir- } \\
\text { fried (with lamb and tomatoes); cooked with rice or } \\
\text { baked with cheese (GR); boiled in mixtures; boiled } \\
\text { alone and consumed with fava bean purée or pork } \\
\text { rinds (IT) }\end{array}$ & $\mathrm{VC}$ & $\begin{array}{l}\text { Depurative, "good for the } \\
\text { liver", against constipation, } \\
\text { strengthening (IT); diuretic, } \\
\text { anti-obesity (GR) }\end{array}$ \\
\hline $\begin{array}{l}\text { Cynara cardunculus L. } \\
\text { (Asteraceae) }\end{array}$ & Agria agkinára (GR) & $\begin{array}{l}\text { Flower } \\
\text { receptacles }\end{array}$ & March-May & $\begin{array}{l}\text { Boiled or cooked in different ways such as with } \\
\text { cultivated artichokes }\end{array}$ & C & Cleaning properties \\
\hline $\begin{array}{l}\text { Melissa officinalis L. } \\
\quad \text { (Lamiaceae) }\end{array}$ & Lemonochorto (GR) & Leaves & All year long & Seasoning in many dishes & C & None \\
\hline $\begin{array}{l}\text { Crepis apula (Fiori) Babc., C. sancta (L.) } \\
\text { Bornm., C. vesicaria L. and possibly } \\
\text { other C. spp. (Asteraceae) }\end{array}$ & $\begin{array}{l}\text { Cannazzicule, Fogghia duce, } \\
\text { Zicumaci (IT) }\end{array}$ & Basal rosettes & February-May & Boiled in mixtures; boiled and then fried & $\mathrm{VC}$ & None \\
\hline $\begin{array}{l}\text { Crepis setosa Haller } \mathrm{f} . \\
\text { (Asteraceae) }\end{array}$ & $\begin{array}{l}\text { Fogghia ndurante, } \\
\text { Miristichella, Miristichiedda, } \\
\text { Mistichiedda (IT) }\end{array}$ & Basal rosettes & February-April & Boiled in mixtures; boiled and then fried & $\mathrm{VC}$ & None \\
\hline $\begin{array}{l}\text { Diplotaxis tenuifolia }(\mathrm{L} .) \text { DC. } \\
\text { (Brassicaceae) }\end{array}$ & $\begin{array}{l}\text { Agria róka (GR); Rugghietta, } \\
\text { Rugula (IT) }\end{array}$ & $\begin{array}{l}\text { Young tender } \\
\text { leaves }\end{array}$ & May-September & $\begin{array}{c}\text { Raw and salty pies (GR); salads, boiled, boiled and } \\
\text { then fried (sometimes on home-made orecchiette } \\
\text { noodles) (IT) }\end{array}$ & $\begin{array}{l}\mathrm{C}(\mathrm{GR}) ; \mathrm{VC} \\
\quad(\mathrm{IT})\end{array}$ & None \\
\hline $\begin{array}{c}\text { Erodium cicutarium (L.) L'Hér. } \\
\text { (Geraniaceae) }\end{array}$ & Chtenáki (GR) & $\begin{array}{l}\text { Tender leaves, } \\
\text { young shoots }\end{array}$ & March-May & $\begin{array}{l}\text { Raw, boiled, and stir-fried with other wild greens } \\
\text { (sometimes with rice or bulgur) }\end{array}$ & $\mathrm{R}$ & Anti-haemorrhagic \\
\hline
\end{tabular}


Table 1. Cont.

\begin{tabular}{|c|c|c|c|c|c|c|}
\hline Botanical taxon (-taxa) and family & Local name(s) & Used plant parts & Gathering time & Traditional culinary use(s) & $\begin{array}{c}\text { Frequency of } \\
\text { quotation }\end{array}$ & $\begin{array}{l}\text { Perceived nutraceutical } \\
\text { property (-ies) }\end{array}$ \\
\hline $\begin{array}{l}\text { Foeniculum vulgare } \mathbf{L} . \\
\text { (Apiaceae) }\end{array}$ & $\begin{array}{c}\text { Fanocchiu restu, Finucchiastru, } \\
\text { Màlaffro (IT); Agrio máratho, } \\
\text { Málathro (GR) }\end{array}$ & $\begin{array}{l}\text { Young aerial } \\
\text { parts (GR \& IT); } \\
\text { inflorescences } \\
\text { and fruits (IT) }\end{array}$ & $\begin{array}{l}\text { March-May (aerial } \\
\text { parts); September } \\
\quad \text { (fruits) }\end{array}$ & $\begin{array}{c}\text { Aerial parts: raw and boiled (GR); filling for home- } \\
\text { made potato pie ("pitta di patate") and for focaccia } \\
\text { bread (IT). Inflorescences: pickled in vinegar, then } \\
\text { eaten in salads (IT). Fruits: seasoning for many } \\
\text { dishes, esp. Muscari-based dishes and home-made } \\
\text { liquors (IT) }\end{array}$ & $\mathrm{VC}$ & $\begin{array}{l}\text { Digestive (GR \& IT); diuretic } \\
\text { (IT) }\end{array}$ \\
\hline $\begin{array}{l}\text { Humulus lupulus L. } \\
\text { (Cannabaceae) }\end{array}$ & Chortaromagià (GR) & $\begin{array}{c}\text { Female } \\
\text { inflorescences }\end{array}$ & May & $\begin{array}{l}\text { Added as a yeasting agent in sourdough bread and } \\
\text { in milk as a yogurt starter }\end{array}$ & $\mathrm{VC}$ & None \\
\hline $\begin{array}{l}\text { Lactuca serriola } \mathrm{L} . \\
\quad \text { (Asteraceae) }\end{array}$ & $\begin{array}{l}\text { Agriomàroylo, Galatsída (GR); } \\
\text { Marie, Marìule (IT) }\end{array}$ & Basel rosettes & $\begin{array}{l}\text { February-April (GR); } \\
\text { March-May (IT) }\end{array}$ & $\begin{array}{l}\text { Boiled (GR); boiled in mixtures; boiled and then } \\
\text { fried (IT) }\end{array}$ & C & None \\
\hline $\begin{array}{l}\text { Laurus nobilis L. } \\
\quad \text { (Lauraceae) }\end{array}$ & Dafni (GR); Lauru (IT) & Leaves & July & Seasoning for many dishes & $\begin{array}{l}\text { C (GR); R } \\
\quad \text { (IT) }\end{array}$ & $\begin{array}{l}\text { Cleansing, anti-obesity (GR); } \\
\text { digestive (IT) }\end{array}$ \\
\hline $\begin{array}{l}\text { Malva sylvestris L. } \\
\quad \text { (Malvaceae) }\end{array}$ & $\begin{array}{l}\text { Meloca, Marva, Panettieddhu } \\
\text { (only the fruits) (IT); Molocha } \\
\text { (GR) }\end{array}$ & $\begin{array}{l}\text { Tender leaves } \\
\text { and fruits }\end{array}$ & $\begin{array}{l}\text { March-May (GR); } \\
\text { February-March } \\
\text { (IT) (leaves); July- } \\
\text { September (fruits) }\end{array}$ & $\begin{array}{l}\text { Leaves: boiled, salty pies, and soups (GR); boiled in } \\
\text { mixtures and omelettes (IT). Fruits: eaten raw as a } \\
\text { snack, esp. by kids (fruits) (GR \& IT) }\end{array}$ & C & None \\
\hline $\begin{array}{l}\text { Mentha spp. } \\
\text { (Lamiaceae) }\end{array}$ & Agria menta (GR) & Leaves & March-September & Seasoning for many dishes and liquors & $\mathrm{VC}$ & $\begin{array}{l}\text { Digestive, liver cleansing and } \\
\text { anti-obesity }\end{array}$ \\
\hline $\begin{array}{l}\text { Muscari comosum (L.) Mill. } \\
\text { (Hyacinthaceae) }\end{array}$ & $\begin{array}{l}\text { Lampascione, Lampone, } \\
\text { Pampasciune (IT) }\end{array}$ & Bulbs & January-April & $\begin{array}{l}\text { Boiled, left to rest one day in water, then fried } \\
\text { and possibly coupled with pork meat, eggs, cherry } \\
\text { tomatoes, and wild fennel (typically with a side } \\
\text { dish of beans with black olives); roasted, fried in } \\
\text { batter, and filling for focaccia bread }\end{array}$ & $\mathrm{VC}$ & Anti-mouth ulcers \\
\hline $\begin{array}{l}\text { Ocimum basilicum L. } \\
\text { (Lamiaceae)* }\end{array}$ & Vasilikós (GR) & Leaves & All year long & Yeast for sourdough bread & C & $\begin{array}{l}\text { "Good for the brain", appetite } \\
\text { stimulant, and digestive }\end{array}$ \\
\hline $\begin{array}{l}\text { Origanum vulgare } \mathbf{L} . \\
\text { (Lamiaceae) }\end{array}$ & Agria rígani (GR); Rigani (IT) & $\begin{array}{l}\text { Flowering aerial } \\
\text { parts }\end{array}$ & June-September & Seasoning for many dishes & $\mathrm{VC}$ & Digestive (IT) \\
\hline $\begin{array}{l}\text { Orobanche crenata Forssk. } \\
\text { (Orobanchaceae) }\end{array}$ & Spurchia (IT) & Young shoots & February-April & Boiled, boiled and then fried, and roasted & C & None \\
\hline
\end{tabular}


Wild vegetables do not lie: Comparative gastronomic ethnobotany and ethnolinguistics on the Greek traces of the Mediterranean Diet of southeastern Italy

Table 1. Cont.

\begin{tabular}{|c|c|c|c|c|c|c|}
\hline Botanical taxon (-taxa) and family & Local name(s) & Used plant parts & Gathering time & Traditional culinary use(s) & $\begin{array}{c}\text { Frequency of } \\
\text { quotation }\end{array}$ & $\begin{array}{l}\text { Perceived nutraceutical } \\
\text { property (-ies) }\end{array}$ \\
\hline $\begin{array}{l}\text { Papaver rhoeas } \mathrm{L} . \\
\text { (Papaveraceae) }\end{array}$ & $\begin{array}{l}\text { Koytsoynada, Paparoyna (GR); } \\
\text { Paparina (IT) }\end{array}$ & Basal rosettes & January-March & $\begin{array}{c}\text { Boiled, stir-fried with onions, lamb and tomatoes, } \\
\text { and soups (GR); boiled and/or fried and as focaccia- } \\
\text { bread filling (IT) }\end{array}$ & VC & Laxative (IT) \\
\hline $\begin{array}{l}\text { Picris hieracioides Sibth. \& Sm. } \\
\text { (Asteraceae) }\end{array}$ & Spruscinu (IT) & Basal rosettes & January-April & Boiled in mixtures, then possibly fried; soups & $\mathrm{VC}$ & None \\
\hline $\begin{array}{l}\text { Pisum sativum ssp. elatius (M.Bieb.) } \\
\text { Asch. \& Graebn. (Fabaceae) }\end{array}$ & Piseddhu restu (IT) & Seeds & April-May & Boiled/cooked as the cultivated relative & $\mathrm{R}$ & None \\
\hline $\begin{array}{l}\text { Pleurotus eryngii (DC.) Quél. } \\
\text { (Pleurotaceae) }\end{array}$ & Cardunceddhu (IT) & Fruiting body & $\begin{array}{l}\text { March-May and } \\
\text { October-December }\end{array}$ & Fried with tomatoes, garlic and chili & C & None \\
\hline $\begin{array}{l}\text { Portulaca oleracea L. } \\
\quad \text { (Portulacaceae) }\end{array}$ & $\begin{array}{l}\text { Glistrida, Antracla (GR); } \\
\text { Andracla, Brucacchia (IT) }\end{array}$ & Aerial parts & June-September & Salads, boiled, and pickled (GR \& IT) & VC & $\begin{array}{l}\text { Anti-ulcers, strengthening, } \\
\text { and appetite stimulant (GR); } \\
\text { laxative, diuretic, and good } \\
\text { "for health" (IT) }\end{array}$ \\
\hline $\begin{array}{l}\text { Rapistrum rugosum (L.) All. } \\
\text { (Brassicaceae) }\end{array}$ & Rapeste, Rapiste, Sanapi (IT) & $\begin{array}{l}\text { Young aerial } \\
\text { parts }\end{array}$ & March-April & Boiled in mixtures; boiled and then fried & C & None \\
\hline $\begin{array}{l}\text { Reichardia picroides (L.) Roth. } \\
\text { (Asteraceae) }\end{array}$ & $\begin{array}{l}\text { Galatsída (GR); Caccialepri, } \\
\text { Cacciareculi, Cazzareculi (IT) }\end{array}$ & Basal rosettes & November-March & $\begin{array}{l}\text { Boiled (GR); raw on bread and in boiled mixtures } \\
\text { (IT) }\end{array}$ & $\begin{array}{l}\mathrm{R}(\mathrm{GR}) ; \mathrm{C} \\
\quad(\mathrm{IT})\end{array}$ & None \\
\hline $\begin{array}{l}\text { Rhagadiolus stellatus (L.) Gaertn. } \\
\text { (Asteraceae) }\end{array}$ & $\begin{array}{l}\text { Lattucheddra, Latturia, } \\
\text { Lattusedda (IT) }\end{array}$ & Basal rosettes & March-April & Boiled in mixtures; boiled and then fried & $\mathrm{R}$ & None \\
\hline $\begin{array}{l}\text { Rumex crispus L. and other Rumex } \\
\text { spp. (Polygonaceae) }\end{array}$ & $\begin{array}{l}\text { Agriosesklo, Lapata, Lapato, } \\
\text { Lapatoydia, Xinitra (GR); } \\
\text { Lapazzu, Rapazzu (IT) }\end{array}$ & Leaves & September-March & $\begin{array}{l}\text { Boiled, salty pies, soups, rolled and baked with } \\
\text { cheese inside, and pickled (GR); boiled, boiled and } \\
\text { fried (IT) }\end{array}$ & $\mathrm{VC}$ & $\begin{array}{l}\text { Anti-obesity, liver cleansing, } \\
\text { and digestive (GR) }\end{array}$ \\
\hline $\begin{array}{l}\text { Scandix pecten-veneris } \mathrm{L} \text {. } \\
\text { (Apiaceae) }\end{array}$ & Myroni (GR) & Young leaves & March-May & Seasoning (esp. in soups) & $\mathrm{R}$ & None \\
\hline $\begin{array}{l}\text { Silybum marianum L. Gaertn. } \\
\text { (Asteraceae) }\end{array}$ & $\begin{array}{l}\text { Agrio gaidoyragkatho (GR); } \\
\text { Crattaluri, Lattaruni (IT) }\end{array}$ & $\begin{array}{l}\text { Basal rosettes, } \\
\text { leaves, and stems } \\
\text { (after removing } \\
\text { thorns) }\end{array}$ & October-April & $\begin{array}{l}\text { Salads, boiled, boiled and then filling in salty pies } \\
\text { (GR); soups, boiled and then fried, boiled and then } \\
\text { fried and baked in the oven (IT) }\end{array}$ & $\begin{array}{l}\mathrm{R}(\mathrm{GR}) ; \mathrm{VC} \\
\quad \text { (IT) }\end{array}$ & Strengthening \\
\hline
\end{tabular}


Table 1. Cont.

\begin{tabular}{|c|c|c|c|c|c|c|}
\hline Botanical taxon (-taxa) and family & Local name(s) & Used plant parts & Gathering time & Traditional culinary use(s) & $\begin{array}{c}\text { Frequency of } \\
\text { quotation }\end{array}$ & $\begin{array}{l}\text { Perceived nutraceutical } \\
\text { property (-ies) }\end{array}$ \\
\hline $\begin{array}{l}\text { Sinapis alba L. and S. arvensis L. } \\
\text { (Brassicaceae) }\end{array}$ & $\begin{array}{l}\text { Agrio sinapi, Pirpiroyna, } \\
\text { Vroyva (GR); Rapesta, Sanapu, } \\
\text { Sinapa (IT) }\end{array}$ & $\begin{array}{l}\text { Young leaves and } \\
\text { seeds }\end{array}$ & March-June & $\begin{array}{l}\text { Young leaves: boiled and in salty pies; boiled in } \\
\text { mixtures (IT). } \\
\text { Seeds: as a natural preservative for wine (GR) }\end{array}$ & $C$ & None \\
\hline $\begin{array}{l}\text { Smilax aspera L. } \\
\quad(\text { Smilaceae })\end{array}$ & Scrasciacane, Viticedda (IT) & Young shoots & February-March & Boiled; boiled and then pickled & C & None \\
\hline $\begin{array}{l}\text { Smyrnium olusatrum L. } \\
\text { (Apiaceae) }\end{array}$ & $\begin{array}{l}\text { Agrioselino (GR); Lacciu crestu, } \\
\text { Svermi, Sverni, Zavirna (IT) }\end{array}$ & $\begin{array}{l}\text { Young shoots } \\
\text { and fleshy } \\
\text { sprouts }\end{array}$ & November-April & $\begin{array}{l}\text { Boiled, salty pies, and seasoning (GR); boiled in } \\
\text { mixtures; boiled and then fried; roasted, on pasta, } \\
\text { boiled and then fried in egg-batter at special } \\
\text { holidays (St. Martin in November, and during } \\
\text { Christmas and Carnival) (IT) }\end{array}$ & $\begin{array}{l}\text { VC in one } \\
\text { village only; } \\
\text { R elsewhere } \\
\text { (IT); R (GR) }\end{array}$ & None \\
\hline $\begin{array}{l}\text { Sonchus asper L. Hill., S. oleraceus } \\
\text { (L.) L., and S. tenerrimus L. } \\
\text { (Asteraceae) }\end{array}$ & $\begin{array}{c}\text { Tsalia, Zochakia, Zochos (GR); } \\
\text { Zangune (IT) }\end{array}$ & Basal rosettes & February-April & $\begin{array}{l}\text { Boiled, boiled and fried, salty pies (GR); boiled in } \\
\text { mixtures, boiled and then fried (IT) }\end{array}$ & $\mathrm{VC}$ & $\begin{array}{l}\text { Anti-anaemia, blood and lever } \\
\text { cleansing, strengthening (GR); } \\
\text { digestive, strengthening (IT) }\end{array}$ \\
\hline Taraxacum spp. (Asteraceae) & Pikralida, Galatsida (GR) & Basal rosettes & October-April & Boiled and in salty pies (GR) & $\mathrm{VC}$ & None \\
\hline $\begin{array}{c}\text { Thymbra capitata (L.) Cav. } \\
\text { (Lamiaceae) }\end{array}$ & $\begin{array}{l}\text { Thymari (GR); Tuni, Tunu } \\
\text { (IT) }\end{array}$ & Aerial parts & All year long & Seasoning for many dishes & $\begin{array}{l}\mathrm{VC}(\mathrm{GR}) ; \mathrm{C} \\
\quad(\mathrm{IT})\end{array}$ & None \\
\hline Tordylium apulum L. (Apiaceae) & Kaykalida, Kaykalithra (GR) & Basal rosettes & February-March & Boiled and in salty pies (GR) & $\mathrm{VC}$ & None \\
\hline $\begin{array}{c}\text { Urospermum picroides L. Scop. ex } \\
\text { F.W.Schmidt } \\
\text { (Asteraceae) }\end{array}$ & Cicoreddha duce (IT) & Basal rosettes & January-April & Boiled in mixtures and soups & $\mathrm{R}$ & None \\
\hline $\begin{array}{l}\text { Urtica dioica } \mathrm{L} . \\
\text { (Urticaceae) }\end{array}$ & $\begin{array}{l}\text { Tsoykneda (GR); Ardica, } \\
\text { Ardicula, Urdicula (IT) }\end{array}$ & $\begin{array}{l}\text { Young aerial } \\
\text { parts }\end{array}$ & January-April & $\begin{array}{l}\text { Boiled, salty pies, and soups (with potatoes and } \\
\text { onions or just flour) (GR); salads, boiled and then } \\
\text { stir-fried, omelettes, and risotto (IT) }\end{array}$ & $\begin{array}{l}\mathrm{VC}(\mathrm{GR}) ; \mathrm{R} \\
\quad \text { (IT) }\end{array}$ & Strengthening (GR) \\
\hline
\end{tabular}

*: cultivated species

GR: NE Greek study site

IT: SE Italian study site 
1964; Porphyrius 1982): Asparagus (aspáragoi), Foeniculum (marathon), Lactuca (amarullion, marulion), Malva (malachīs), Origanum (origanon), Portulaca (andrachnī), and Sonchus (sonchos); and possibly also for Crepis setosa (myrìzō; see following paragraph). These wild vegetables may represent a portion of an ancient Greek ethnobotanical culinary heritage that migrated to Southern Italy many centuries ago.

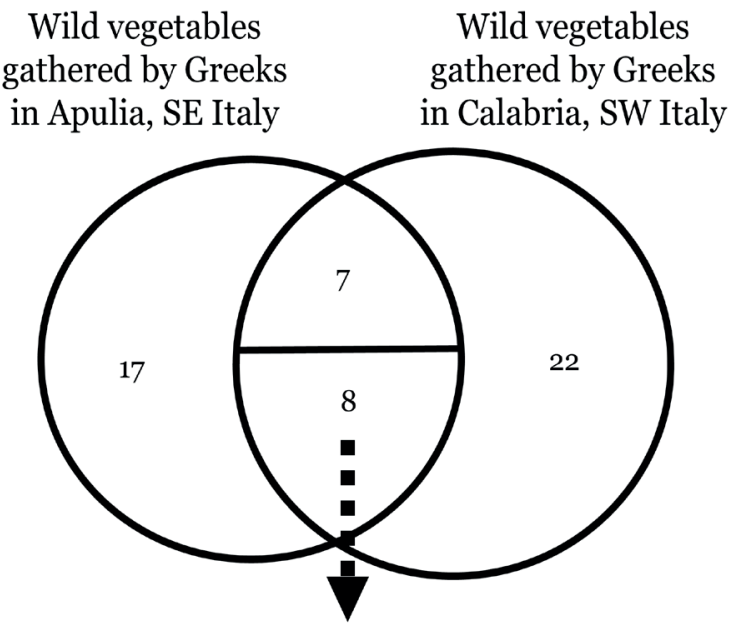

Species named by Greek cognates

Figure 4. Comparison of the wild vegetables (botanical species) gathered by the Griko people in SE Italy and the Graecanic diaspora in SW Italy (Nebel et al. 2006).

\section{Discussion}

\section{Griko culinary uses of wild vegetables}

All of the interviewees at both sites normally collected wild greens in and around cultivated fields where they were certain that no chemicals had been used on the land. Although both women and men typically collected wild greens, women were responsible for cooking them.

The case of Muscari comosum in Apulia is particularly interesting as it is collected by men only. The main reason for this is that its location underground makes extraction very laborious. Some of the men recalled that their mothers and grandmothers used to reward them with a little money for every bulb brought home and that this was their 'initiation' to wild greens.

Culinary 'manipulations' of the gathered wild greens are different between the two study areas. The most frequently recorded SE Italy traditional culinary preparations include two mixtures of wild greens, which are even sometimes sold in 'informal' street markets (see Fig. 5): misticanza (boiled mixtures of wild vegetables): multiple species of wild greens are boiled together and then dressed with extra virgin olive oil and salt; lemon and/or cheese are optional; minestra (boiled and fried mixtures of wild vegetables): multiple species of wild greens are parboiled. If they are young and very tender, this step is skipped. Next, the greens are stir-fried with extra virgin olive oil and either garlic or onion and sometimes additional ingredients, such as fresh cherry tomatoes, pork, hard cheeses (e.g. Pecorino) and chili peppers. They can be placed in layers in a pot and baked in an oven. This way of cooking was likely born out of the need to make use of leftovers. It also provided a way to preserve the wild greens for a longer time.

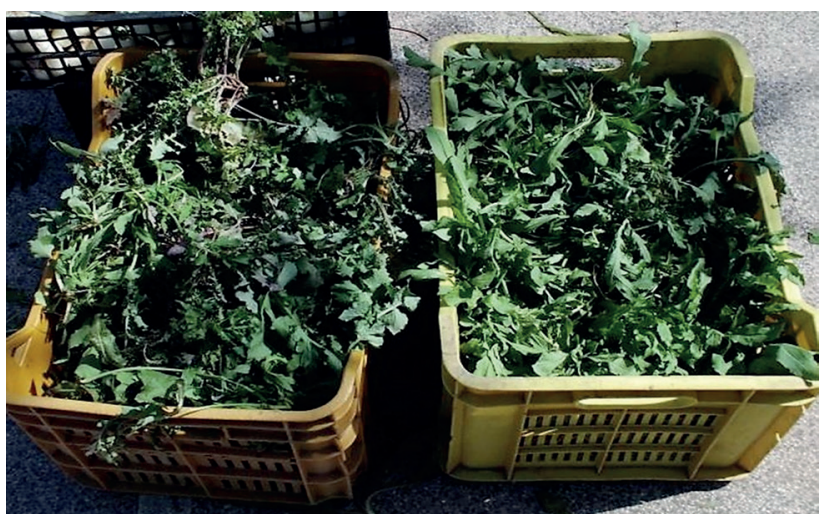

Figure 5. Combinations of wild vegetables sold in informal street markets (SE Italy).

In SE Italy we also recorded recipes for some species (six folk taxa) that were specific to the area: Papaver rhoeas (paparina) is always stir-fried with extra virgin olive oil, garlic, chili peppers and black olives. Moreover, this plant is often cooked in combination with Rumex spp. (lapazzu). In the South Italian dialect spoken in the study area, there were even folk songs based on this style of preparation: 'Nazzu nazzu nazzu la paparina cu lu lapazzu ca senza lu lapazzu la paparina ci me lu fazzu?' ('What can I do with paparina if I do not have the lapazzu?'); pittule ripiene is a common dish in Salento, usually prepared for special occasions, such as Christmas or Carnival. It consists of deep-fried leavened rolls, usually made with fillings, such as young shoots of Smyrnium olusatrum or Borago officinalis; the young inflorescences of wild Foeniculum vulgare (also known as caruselle) (see Fig. 6) are slightly boiled and then preserved in vinegar for later use in salads or as a filling for potato pies; cardi racanati is a dish in which the main ingredients are the leaves, leaf stalks and stems, previously cleaned of thorns, of wild Carduus and Galactites ssp. (see Fig. 7). First, the aforementioned ingredients are quickly parboiled and stir-fried with extra virgin olive oil, spring onions and cherry tomatoes; they are then layered with cheese and breadcrumbs in a pot and baked in an oven; Cichorium intybus (Cicureddha) is perfectly suited for the fava bean purée that distinguishes the popular 'fave e cicorie' dish.

\section{NE Greek culinary uses of wild vegetables}

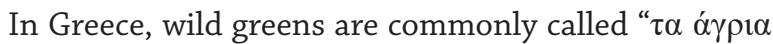

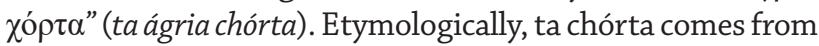




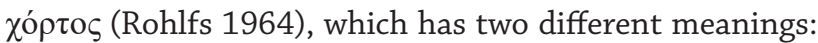
'grassland' and the widely used 'fodder, hay or herb'. In

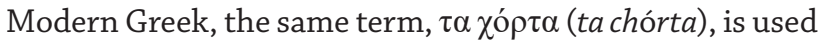
to refer to the wild greens collected and eaten by the local people. According to an analysis by Nebel \& Heinrich (2009), there has possibly been a fascinating change in meaning from 'animal fodder' in Ancient Greece to today's 'herbs for human consumption'.
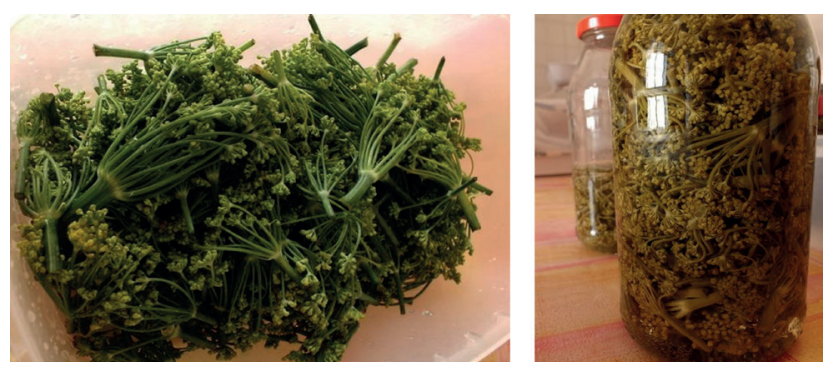

Figure 6. A speciality food of the Griko cuisine: inflorescences of wild fennel (caruselle) before and after being pickled in vinegar.

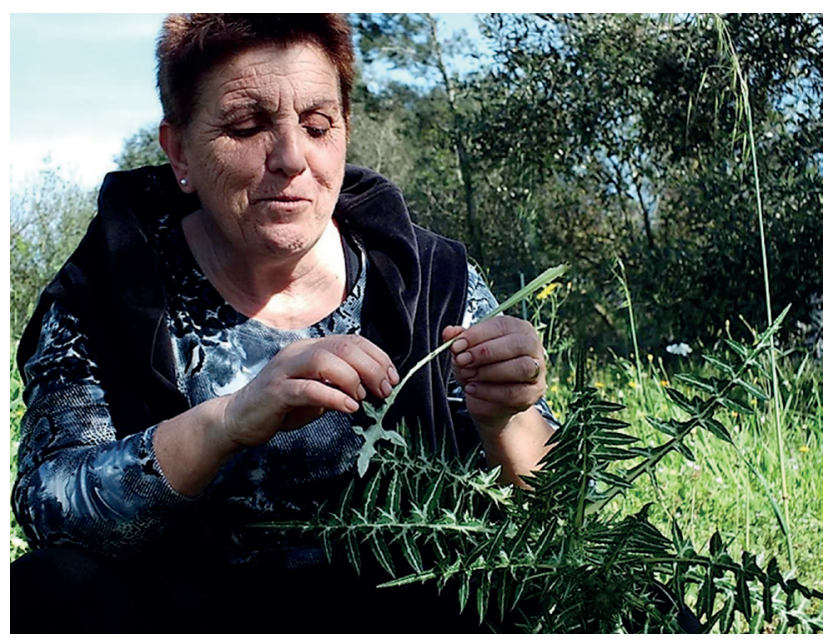

Figure 7. A Griko woman removing thorns from Galactitis tomantosa leaf stalks.

The most common culinary uses of wild vegetables recorded in NE Greece in this study were the following: $\sigma \alpha \lambda \alpha \dot{\tau} \alpha \alpha$ (saláta): the herbs are parboiled (or boiled if very tough and bitter) and then dressed with extra virgin olive

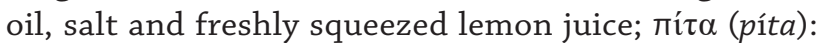
common to all former Ottoman territories, this is a typical Greek pie made with phyllo dough and fillings such as cheese, meat, and vegetables with cheese. A particular example is $\gamma \kappa о \lambda ı \mu \pi \lambda i v \alpha$ (gkoliomplina), meaning 'naked', which is a pie made without phyllo dough; instead, a dough made of lard and flour is used. This type of pie is usually filled with Chenopodium album; fricassée: this is made with lamb or goat meat stewed in a pan with onions, wild greens and, sometimes, a few aromatic herbs. The study participants typically dressed this with a sauce made with eggs, lemon juice and a small amount of the juices from the cooked meat before serving it; soups: these are prepared with water and corn flour, grains, such as rice or bulgur, or potatoes. Lemon juice and egg sauce are normally added at the end. Of the specific soups, two are worth mentioning: $\mu \alpha \gamma \varepsilon i \rho i ́ \tau \sigma \alpha$ (mageiritsa) with lamb, onions, lettuce, Anthriscus or Scandix spp., lemon and egg

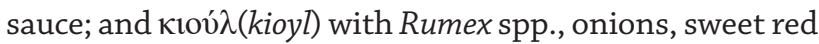
peppers, flour and water.

Aromatic herbs, such as Foeniculum or the Mentha spp., are generally added to many NE Greece dishes, especially $v \tau 0 \lambda \mu \alpha \delta \dot{\alpha} \kappa ı \alpha$ (ntolmadákia), the characteristic rice-filled vine leaf rolls.

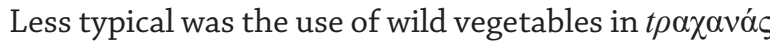

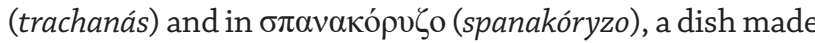
with rice boiled together with spinach or, sometimes, the tender shoots of Urtica dioica. The former is an ancient dish found throughout the Balkan region. In the NE Greece

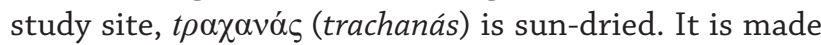
from crushed wheat and fermented milk to which stock or water is added to make a thick soup. It can be either savoury, with the addition of dried vegetables, or sweet, with dried fruits.

\section{Continuity and change in ancient 'Greek' and Near East uses of wild vegetables}

The frequency of the consumption of wild vegetables in NE Greece is much higher than that in SE Italy even though the study region in Italy is considered the national 'lynchpin' of traditional plant foraging (Biscotti et al. 2018). This suggests that many of the original features associated with the wild vegetable-based folk cuisine of the Griko diaspora might have been lost after the move west.

Within the SE Italy study site, very few vegetables, such as Diplotaxis, Portulaca and the Reichardia spp., were traditionally consumed raw; however, in NE Greece, this was the case for wild Allium, Carduus, Cichorium, Erodium, Foeniculum and Portulaca spp. Raw consumption is an ancient practice that might have its origin in 'ethnobotanical snacks'. This term refers to the raw wild plant parts consumed singly on the spot and not in domestic settings. In other words, no ingredients were added, and the plant parts were consumed at the location in which they were found. It could be that as time passed, they were gathered and eaten on simple breads in the home. The consumption of raw Calystegial Convolvulus spp. as a 'medicinal/digestive' snack might have an ancient origin that was likely lost when the use of this folk taxon migrated to Southern Italy, where the same group of species had been ignored by locals.

A similar ancient origin can be ascribed to the raw onthe-spot consumption of Carduus spp., a practice still seen in NE Greece but not in the SE Italian site. This might represent one of the elements of the complex raw dietary system of wild thistles and related species of possible pastoralist origin. The same system might also be responsible for the later domestication of the artichoke. 
Additionally, a similar use among the Yazidis of Iraqi Kurdistan (Pieroni et al. 2018) suggests that the consumption of raw Erodium spp. could have an Eastern or a Near East origin; it is possible that the practice might have been lost when Greeks migrated west to SE Italy.

The descendants of the old Mesopotamian Assyrians in Iraq were recently recorded (Pieroni et al. 2018) as having a post-Neolithic weedy wild vegetable-centred food system based on wild Asteraceae and, to a lesser extent, Brassicaceae that grow around cultivated fields. This diet seems to be very similar to that recorded at the two study sites. In SE Italy and NE Greece, a remarkable number of bitter and/ or pungent taxa were boiled, and in SE Italy, they were sometimes boiled and then fried.

A comparison of the data collected from the two sites and the locations of the other Greek diasporas in Italy showed that a dozen weedy species, frequently mentioned in both SE and NE Greece by cognates (see the taxa in bold type in Fig. 1), emerged as 'the core' of a possible ancient, postNeolithic 'synanthropic' Mediterranean Diet: Cichorium, Foeniculum, Lactuca, Malva, Papaver, Portulaca, Sinapis and Sonchus spp.

A review of the Italian food ethnobotanical literature indicates that the consumption of Malva, Papaver and Portulaca aerial parts in Italy, in particular, is very pronounced in the South of the country. Malva and Portulaca have traditionally been exclusive to former Magna Grecia areas: Apulia, Campania, Calabria and Sicily (Guarrera 2007; Pieroni 2017), while the consumption of pickled wild Foeniculum vulgare inflorescences (see Fig. 7) seems to be a specific Griko custom.

Most of these taxa are still very commonly used among Christian Assyrians, who consider themselves descendants of Babylonian Assyrians, in both the Syrian-Turkish borderland (Abdalla 2004) and Iraqi Kurdistan (Pieroni et al. 2018). They are the main ingredients in the wild vegetablecentred Greek folk cuisine of Cyprus (Della et al. 2006) and other Greek diasporas in Southern Italy (Nebel et al. 2006). Moreover, this same group of taxa is also commonly used for food in Apulia (Biscotti et al. 2018), Sicily (Licata et al. 2016; Geraci et al. 2018) and other Mediterranean coastal areas (Dogan 2012; Łuczaj et al. 2013; Łuczaj \& Dolina 2015). It can therefore be assumed that this system might have migrated during the post-Neolithic period from the Near East to Central and Western Mediterranean regions and that Greece might have represented an important stop along the way.

The differences observed in the data related to the Griko and other Greek diasporas in Calabria, SW Italy (only about one-third of all taxa are common to all areas) could be the result of more complex acculturation processes that the latter ones went through and which hybridised the original Greek wild plant uses with those of the autochthonous (Calabrian) populations. Another possible explanation may be related to the fact that SE Italy and Greece are extremely close geographically; thus, the Griko people in SE Italy might have had more regular contact with their home country than did the Graecanic groups of Calabria.

In SE Italy, a few other wild food herbs (Asparagus, Origanum and Thymus spp.) that have Greek names and were commonly quoted in the study areas in both Italy and Greece were not synanthropic. Instead, they likely represent further retentions of a kind of pre-Neolithic 'gathering from the wild' activity, which, especially in the case of Origanum and Thymus spp., might have also had a medicinal origin.

Moreover, the consumption of Smilax aspera and Smyrnium olusatrum shoots, which was recorded as being typical in SE Italy only, could be ascribed to the same preNeolithic 'gathering from the wild' phenomenon. The consumption of these shoots is very rare in other parts of Italy; however, the culinary use of this species is still very popular in some parts of the Caucasus (R Sõukand, A Pieroni unpubl. res) and Kurdistan (Pieroni et al. 2018).

Of particular interest is the case of Crepis setosa, known as miristichella or miristichiedda, which are both lexemes unrelated to the Apulian (South Italian) dialect. This specific taxon was not used in NE Greece; however, the Griko folk name might have originated from the Greek $\mu v p i \zeta \omega$ (myrìzō), which in Modern Greek means 'to smell' but in ancient Greek meant 'to scatter with balm' or 'to perfume'. The other vernacular name for this wild plant recorded in the Italian study site might confirm this hypothesis. Indeed, another term that is sometimes used in SE Italy is the South-Italian erba 'ndurante, which literally means 'long lasting herb', referring to the aroma that permeates the hands once the leaves have been touched. Thus, the enduring scent left by the plant is the key to understanding its name. The phytonyms for this wild vegetable have a Greek origin, but the folk use in NE Greece has probably been lost.

Finally, for the Greek site, the results show that it is quite common to start the fermentation of sour dough or milk to yogurt with the female inflorescences of the wild hop (Humulus lupulus). The use of this ingredient, as well as basil, a commonly utilised plant in Orthodox Christian rites, as yeast could be also related to a ritual meaning ascribed to each of these plants. Similar practices of using hop inflorescences as yeast in the baking of bread, common in Romania until only a few decades ago (Pieroni et al. 2012), are still being observed by an ancient, very isolated Molokan (Russian) diaspora in Azerbaijan in the Caucasus Mountains (A Pieroni, R Sõukand unpubl. res.).

Additionally, more than half of the quoted vegetables were perceived in both study sites as "medicinal foods", thus confirming the fact that Mediterranean rural classes were (and still partially are) used to eating wild plant ingredients to improve general health or even to treat specific diseases (Pieroni et al. 2002; Pieroni \& Quave 2006; Guarrera \& Savo 2013; Cucinotta \& Pieroni 2018). 


\section{Conclusions}

In sum, this study confirmed that cross-cultural comparative ethnobotanical and ethnolinguistic data can be used to trace the trajectories of plant use in ancient times. Such holistic bio-cultural analyses in botany are essential for understanding the temporal and spatial dynamics of plant uses. Any list of plants that are used by a community should be supported by in-depth cross-cultural comparisons and linguistic analyses.

It is important that future studies on the spatial continuum between the Near East and the Western Mediterranean, especially along the Near Eastern, Greek and Northern African coasts, address the ethnobotany and ethnolinguistics of wild vegetables to better assess the continuity and change in the post-Neolithic edible weedbased system that is one of the most significant but still little-known aspects of the Mediterranean Diet.

\section{Acknowledgements}

Special thanks are due to all the study participants in both sites; and to the University of Gastronomic Sciences for having partially funded the study.

\section{References}

Abdalla M. 2004. Wild growing plants in the cuisine of modern Assyrians in the Eastern Syrian-Turkish borderland. Journal of Assyrian Academic Studies 18: 50-58.

Accogli R, Medagli P. 2014. Erbe spontanee salentine. Guida al riconoscimento e all'uso delle piante alimentari tradizionali. Lecce, Edizioni Grifo.

Biscotti N. 2012. Botanica delle erbe eduli. Peregrinazioni fitoalimurgiche dal Gargano alle Puglie. Foggia, Centro Grafico.

Biscotti N, Bonsanto D, Viscio G. 2018. The traditional food use of wild vegetables in Apulia (Italy) in the light of Italian ethnobotanical literature. Italian Botanist 5: 1-24.

Biscotti N, Pieroni A. 2015. The hidden Mediterranean diet: wild vegetables traditionally gathered and consumed in the Gargano area, Apulia, SE Italy. Acta Societatis Botanicorum Poloniae 84: 3.

Conforti F, Marrelli M, Carmela C, et al. 2011. Bioactive phytonutrients (omega fatty acids, tocopherols, polyphenols), in vitro inhibition of nitric oxide production and free radical scavenging activity of noncultivated Mediterranean vegetables. Food Chemistry 129: 1413-1419.

Conforti F, Perri V, Menichini F, et al. 2012. Wild Mediterranean dietary plants as inhibitors of pancreatic lipase. Phytotherapy Research 26: 600-604.

Cucinotta F, Pieroni A. 2018. "If you want to get married, you have to collect virdura": the vanishing custom of gathering and cooking wild food plants on Vulcano, Aeolian Islands, Sicily. Food, Culture \& Society 21: 539-567.

Della A, Paraskeva-Hadjichambi D, Hadjichambis A. 2006. An ethnobotanical survey of wild edible plants of Paphos and Larnaca countryside of Cyprus. Journal of Ethnobiology and Ethnomedicine 2: 34. doi: 10.1186/1746-4269-2-34

Dimopoulos P, Raus T, Bergmeier E, et al. 2016. Vascular plants of Greece: An annotated checklist. Willdenowia 46: 301-347.

Dioscorides P. 1959. The Greek herbal of Dioscorides. New York, Hafner.

di Tizio A, Euczaj E, Quave CL, et al. 2012. Traditional food and herbal uses of wild plants in the ancient South-Slavic diaspora of
Mundimitar/Montemitro (Southern Italy). Journal of Ethnobiology and Ethnomedicine 8: 21. doi: 10.1186/1746-4269-8-21

Ditonno NM, Lamusta S. 2015. Sapori e aromi di piante e frutti spontanei della Puglia peninsulare. Galatina, Congedo Editore.

Dogan Y. 2012. Traditionally used wild edible greens in the Aegean Region of Turkey. Acta Societatis Botanicorum Poloniae 81: 329-341.

Ferrari R, Rapezzi C. 2011. The Mediterranean diet: a cultural journey. Lancet 377: 1730-1731.

Geraci A, Amato F, Noto G, et al. 2018. The wild taxa utilized as vegetables in Sicily (Italy): a traditional component of the Mediterranean diet. Journal of Ethnobiology and Ethnomedicine 14: 14. doi: 10.1186/ s13002-018-0215-x

Ghirardini MP, Carli M, Vecchio N, et al. 2007. The importance of a taste. A comparative study on wild food plant consumption in twenty-one local communities in Italy. Journal of Ethnobiology and Ethnomedicine 3: 22. doi: 10.1186/1746-4269-3-22

Guarrera PM. 2007. Usi e Tradizioni della Flora Italiana. Medicina Popolare ed Etnobotanica. Roma, Aracne.

Guarrera PM, Savo V. 2013. Perceived health properties of wild and cultivated food plants in local and popular traditions of Italy: a review. Journal of Ethnopharmacology 146: 659-680.

Hadjichambis AC, Paraskeva-Hadjichambi D, Della A, et al. 2008. Wild and semi-domesticated food plant consumption in seven circumMediterranean areas. International Journal of Food Sciences and Nutrition 59: 383-414.

Index Fungorum Partnership. 2018. Index Fungorum. http://www. indexfungorum.org/names/names.asp. 20 Sep. 2018.

ISE - International Society of Ethnobiology. 2008. The ISE Code of ethics. http://www.ethnobiology.net/what-we-do/core-programs/ise-ethicsprogram/code-of-ethics/. 20 Sep. 2018.

Lentini F, Venza F. 2007. Wild food plants of popular use in Sicily. Journal of Ethnobiology and Ethnomedicine 3: 15. doi: 10.1186/1746-4269-3-15

Leonti M, Nebel S, Rivera D, et al. 2006. Wild gathered food plants in the European Mediterranean: a comparative analysis. Economic Botany 60: $130-142$

Licata M, Tuttolomondo T, Leto C, et al. 2016. A survey of wild plant species for food use in Sicily (Italy) - Results of a 3-year study in four Regional Parks. Journal of Ethnobiology and Ethnomedicine 12: 12. doi: 10.1186/s13002-015-0074-7

Local Food-Nutraceuticals Consortium. 2005. Understanding local Mediterranean diets: a multidisciplinary pharmacological and ethnobotanical approach. Pharmacological Research 52: 353-366.

Łuczaj $€$, Dolina K. 2015. A hundred years of change in wild vegetable use in southern Herzegovina. Journal of Ethnopharmacology 166: 297-304.

Łuczaj Ł, Končić MZ, Miličević T, et al. 2013. Wild vegetable mixes sold in the markets of Dalmatia (southern Croatia). Journal of Ethnobiology and Ethnomedicine 9: 2. doi: 10.1186/1746-4269-9-2

Łuczaj $€$, Pieroni A, Tardío J, et al. 2012. Wild food plant use in 21st century Europe: the disappearance of old traditions and the search for new cuisines involving wild edibles. Acta Societatis Botanicorum Poloniae 81: 359-370.

Marouf M, Batal M, Moledor S, et al. 2015. Exploring the practice of traditional wild plant collection in Lebanon. Food, Culture \& Society 18: 355-378.

Marrelli M, Loizzo MR, Nicoletti M, Menichini F, Conforti F. 2014. In vitro investigation of the potential health benefits of wild Mediterranean dietary plants as anti-obesity agents with $\alpha$-amylase and pancreatic lipase inhibitory activities. Journal of the Science of Food and Agriculture 94: 2217-2224.

Menotti A, Puddu PE. 2015. How the seven countries study contributed to the definition and development of the Mediterranean diet concept: a 50 -year journey. Nutrition, Metabolism and Cardiovascular Diseases 25: $245-52$.

Montanari M. 2006. Food is culture. Chichester, Columbia University Press. Moseley C. 2010. Atlas of the world's languages in danger, 3rd edition. Paris, UNESCO Publishing.

Naska A, Trichopoulou A. 2014. Back to the future: the Mediterraenan diet paradigm. Nutrition, Metabolism and Cardiovascular Diseases 24: $216-219$ 


\section{Wild vegetables do not lie: Comparative gastronomic ethnobotany and ethnolinguistics on the Greek traces of the Mediterranean Diet of southeastern Italy}

Nebel S, Heinrich M. 2009. Ta chòrta: A comparative ethnobotanicallinguistic study of wild food plants in a Graecanic area in Calabria, Southern Italy. Economic Botany 63: 78-92.

Nebel S, Pieroni A, Heinrich M. 2006. Ta chòrta: wild edible greens used in the Graecanic area in Calabria, Southern Italy. Appetite 47: 333-342.

Parlangeli O. 1989. Sui dialetti romanzi e romaici del Salento. Galatina, Congedo.

Pellegrino M. 2016. Performing Griko beyond ‘death'. Palaver 5: 137162.

Pieroni A. 2017. Atlante Gastronomico delle Erbe. Bra, Slow Food Editore.

Pieroni A, Quave CL 2006. Functional foods or food medicines? On the consumption of wild plants among Albanians and Southern Italians in Lucania. In: Pieroni A, Price LL. (eds.) Eating and healing: traditional food as medicine. Binghamton, Haworth Press. p. 101-129.

Pieroni A, Quave CL, Giusti ME, et al. 2012. "We are Italians!": the hybrid ethnobotany of a Venetian diaspora in Eastern Romania. Human Ecology 40: 435-451.

Pieroni A, Nebel S, Quave C, et al. 2002. Ethnopharmacology of liakra: traditional weedy vegetables of the Arbëreshë of the Vulture area in southern Italy. Journal of Ethnopharmacology 81: 165-185.

Pieroni A, Nebel S, Santoro R, et al. 2005. Food for two seasons: culinary uses of non-cultivated local vegetables and mushrooms in a south Italian village. International Journal of Food Sciences and Nutrition 56: $245-272$

Pieroni A, Sõukand R, Amin HIM, et al. 2018. Celebrating multi-religious co-existence in Central Kurdistan: the bio-culturally diverse traditional gathering of wild vegetables among Yazidis, Assyrians, and Muslim Kurds. Human Ecology 46: 217-227.

Pignatti S. 1982. Flora d'Italia. Bologna, Edizioni Edagricole.

Porphyrius. 1982. Vie de Pythagore; Lettre à Marcella. Paris, Les Belles Lettres.

Quave CL, Saitta A. 2016. Forty-five years later: The shifting dynamic of traditional ecological knowledge on Pantelleria Island, Italy. Economic Botany 70: 380-393.

Rigat M, Gras A, D’Ambrosio U, et al. 2016. Wild food plants and minor crops in the Ripollès district (Catalonia, Iberian Peninsula): potentialities for developing a local production, consumption and exchange program. Journal of Ethnobiology and Ethnomedicine 12: 49. doi: 10.1186/ s13002-016-0122-y

Rohlfs G. 1964. Lexicon Graecanicum Italiae Inferioris. Etymologisches Wörterbuch der unteritalienischen Gräzität. Tübingen, Niemeyer.

Rohlfs G. 1967. Greek remnants in southern Italy. The Classical Journal 62: 164-169.

Romojaro A, Botella MÁ, Obón C, et al. 2013. Nutritional and antioxidant properties of wild edible plants and their use as potential ingredients in the modern diet. International Journal of Food Sciences and Nutrition 64: 944-952.

Samsaris DC. 1988. Le royaume client thrace aux temps de Tibere et la tutelle romaine de Trebellenus Rufus. Le stade transitif de la clientele a la provincialisation de la Thrace. Dodona 17: 159-168.

Santayana MP, Tardío J, Blanco E, et al. 2007. Traditional knowledge of wild edible plants used in the northwest of the Iberian Peninsula (Spain and Portugal): a comparative study. Journal of Ethnobiology and Ethnomedicine 3: 27. doi: 10.1186/1746-4269-3-27

Stavridakis GK. 2006. Hē agria vrōsimē chlōrida tēs Krētēs - Wild edible plants of Crete. Rethymno, K.G. Stavridakis.

Stevens PF. 2017. Angiosperm Phylogeny Website. Vers. 14. http://www. mobot.org/MOBOT/research/APweb/. 20 Sept. 2018.

Tardío J, Pascual H, Morales R. 2005. Wild food plants traditionally used in the province of Madrid, Central Spain. Economic Botany 59: 122-136.

The Plant List. 2018. The Plant List. http://www.theplantlist.org/. 20 Sep. 2018.

Trichopoulou A, Vasilopoulou E, Hollman P, et al. 2000. Nutritional composition and flavonoid content of edible wild greens and green pies: a potential rich source of antioxidant nutrients in the Mediterranean diet. Food Chemistry 70: 319-323.

Tutin TG, Heywood VH, Burges NA, et al. 2001. Flora Europaea. Cambridge, Cambridge University Press.

UNESCO. 2013. Mediterranean diet. https://ich.unesco.org/en/RL/ mediterranean-diet-00884. 20 Sep. 2018.

Vasilopoulou E, Trichopoulou A. 2011. Green pies: The flavonoid rich Greek snack. Food Chemistry 126: 855-858. 